\title{
Capacity Scaling of Multihop Cellular Networks
}

\author{
Pan $\mathrm{Li}^{*}$, Xiaoxia Huang ${ }^{\dagger}$, and Yuguang Fang ${ }^{\ddagger}$ \\ * Department of Electrical and Computer Engineering, Mississippi State University, MS 39762 \\ †Shenzhen Institutes of Advanced Technology, Chinese Academy of Sciences, Shenzhen, China \\ $\ddagger$ Department of Electrical and Computer Engineering, University of Florida, FL 32611 \\ Email: li@ece.msstate.edu, xx.huang@sub.siat.ac.cn, fang@ece.ufl.edu
}

\begin{abstract}
Wireless cellular networks are large-scale networks in which asymptotic capacity investigation is no longer a cliché. A substantial body of work has been carried out to improve the capacity of cellular networks by introducing ad hoc communications, resulting in the so-called multihop cellular networks. Most of the previous research allows ad hoc transmissions between certain source and destination pairs to alleviate base stations' relay burden. However, since reports show that Internet data traffic is becoming more and more dominant in cellular networks, we explore in this paper the capacity of multihop cellular networks with all traffic going through base stations and ad hoc transmissions only acting as relay. We first investigate the capacity of regular multihop cellular networks where both nodes and base stations are regularly placed. By fully exploiting the link rate variability, we find that multihop cellular networks can have higher per-node throughput than traditional cellular networks by a scaling factor of $\log _{2} n$. Then, for the first time we extend our study to the capacity of heterogeneous multihop cellular networks where nodes are distributed according to a general Inhomogeneous Poisson Process and base stations are randomly placed. We show that under certain conditions multihop cellular networks can also outperform traditional cellular networks by a scaling factor of $\log _{2} n$. Moreover, both throughput-fairness and bandwidth-fairness are considered as fairness constraints for both kinds of networks.
\end{abstract}

\section{INTRODUCTION}

Wireless cellular networks have been widely deployed since 1980s. In a cellular network, every node transmits (or receive) all its traffic to (or from) a base station in one hop, and the throughput for each node is very low. Realizing that ad hoc networks can accommodate more concurrent transmissions and have higher network spatial reuse ratio, Lin et al. [10] and Luo et al. [12] introduce ad hoc communications into cellular networks, resulting in the so-called multihop cellular networks or hybrid wireless networks ${ }^{1}$, where some transmissions are carried out between a node and a base station directly in one hop and the others are performed between two nodes via multiple hops. They show that multihop cellular networks can have higher throughput than traditional cellular networks by presenting some numerical results. However, nowadays cellular networks are getting larger and larger, and the numerical

This work was partially supported by the U.S. National Science Foundation under grants CNS-0916391 and CNS-0721744. The work of Huang was also partially supported by the National Natural Science Foundation of China under grant 60903192 and Guangdong S\&T Major Project under grant 2009A080207002. The work of Y. Fang was also partially supported by the National Natural Science Foundation of China under grant 61003300 and China 111 Project under grant B08038.

${ }^{1}$ In the sequel, we use these two terms interchangeably. results obtained in limited-size networks are insufficient to describe the behavior of such large systems. In particular, according to the CTIA The Wireless Association [3], the number of wireless subscribers in the U.S. is $97 \mathrm{M}$ in $2000,194.4 \mathrm{M}$ in 2005 , and $276.6 \mathrm{M}$ in 2009 , respectively, and the number of cell sites is $95.7 \mathrm{~K}$ in $2000,178 \mathrm{~K}$ in 2005 , and $245.9 \mathrm{~K}$ in 2009 , respectively, which are both keeping increasing dramatically. So obviously we are having large-scale wireless networks today. The asymptotic capacity investigation is thus no longer a cliché, which can better unveil the network performance.

The capacity of hybrid wireless networks has been extensively studied in the literature such as [8], [9], [11], [15], [17]. All these efforts improve the capacity of traditional cellular networks by allowing ad hoc communications between certain sources and destinations without the help of base stations so as to relieve their relay burden. However, due to the emerging various data services, Internet data traffic is becoming more and more dominant, and according to Nokia Siemens Networks [1], Internet data traffic will overtake voice traffic by 2011, and grow exponentially until 2013. As a result, in this paper, we attempt to explore the capacity of multihop cellular networks considering that all traffic goes through base stations and ad hoc transmissions only act as relay. Specifically, we carry out one-hop direct transmissions to or from base stations only for a fraction of nodes close to them. The other nodes farther away need communicate via multiple hops with some of the above nodes in order to communicate with base stations. In so doing, although we still consider randomly chosen source-destination pairs in the network, the obtained capacity results can also account for the case when Internet traffic takes a majority.

The main contribution of this paper is threefold. First, we investigate the capacity of regular multihop cellular networks where both nodes and base stations are regularly placed. By fully exploiting the link rate variability, we try to find whether and how much ad hoc relay can help improve the network capacity. The most related work in the literature is by Law et al. [7], which formulates the throughput of regular multihop cellular networks as an optimization problem and gives some numerical results. The derived throughput in [7] is an upper bound which is not necessarily achievable. While in this work, we obtain for the first time theoretical results on scaling capacity, and what we present is feasible throughput. Second, we extend our study to the capacity of general heterogeneous multihop cellular networks. All the aforementioned 
work is carried out in homogeneous networks where nodes are either regularly or uniformly distributed. Alfano et al. [2] study the capacity of inhomogeneous ad hoc networks with nodes distributed according to a shot-noise Cox process, a special heterogeneous point distribution process, which is quite different from the problem in this study. To the best of our knowledge, this is the first attempt to explore the capacity of heterogeneous multihop cellular networks in which nodes are distributed according to a general inhomogeneous point process. We also explore under what conditions can ad hoc relay improve the network capacity. Third, both throughputfairness and bandwidth-fairness are considered as fairness constraints for both kinds of networks.

The main results of this paper are briefly summarized as follows. We find that by fully taking advantage of link rate variability, regular multihop cellular networks can have higher per-node throughput than traditional cellular networks by a scaling factor of $\log _{2} n$, under either throughput-fairness or bandwidth-fairness constraint. We also show that under certain conditions heterogeneous multihop cellular networks can also outperform traditional cellular networks by a scaling factor of $\log _{2} n$.

The rest of this paper is organized as follows. In Section II we introduce the network model and the channel capacity model we use in this work. Section III and Section IV present the capacity of regular multihop cellular networks and of heterogeneous multihop cellular networks, respectively. We finally conclude this paper in Section V.

\section{Assumptions AND Models}

In this section, we introduce the network model and the channel capacity model we will use throughout this paper.

\section{A. Network Model}

We consider a network with $n$ nodes and $m$ base stations distributed on a two-dimensional torus with edge $L$, where $m=n^{\beta}(0<\beta<1), L=n^{\alpha}(0 \leq \alpha \leq 1 / 2)$, and the network area $|\mathcal{A}|=L^{2}$. Thus, we can model all kinds of networks including dense networks $(\alpha=0)$ [11], extended networks $(\alpha=1 / 2)[8]$, and semi-extended networks $(0<$ $\alpha<1 / 2$ ) [4]. The $m$ base stations are interconnected via a wired network, in which the link bandwidth is large enough and there is no bandwidth constraint.

Regular Multihop Cellular Networks: We first assume that the $n$ nodes and the $m$ base stations are regularly placed in the network, respectively. The $m$ base stations divide the network into sets of hexagons, i.e., cells. At the center of each cell there is a base station.

Heterogeneous Multihop Cellular Networks: We then extend our study to the case when the $n$ nodes are distributed according to a general Inhomogeneous Poisson Process (IPP), with the local intensity at point $\xi$ in the network denoted by $\Psi(\xi)$, and $\int_{\mathcal{A}} \Psi(\xi) \mathrm{d} \xi=n$. The minimum and the maximum of $\Psi(\xi)$ are denoted by $\underline{\Psi}$ and $\bar{\Psi}$, respectively, which both scale with $n$. Assume $\underline{\Psi} n^{2 \alpha}=\omega(\ln n)$. We also assume that the $m$ base stations are uniformly and randomly distributed in the network.

We follow the process in [5] to choose random senderreceiver pairs so that each node is a source node for one flow and a destination node for at most $\mathrm{O}(1)$ flows. The nodes in a cell transmit or receive all packets through the base station in the cell, either in a single hop or across multiple hops. Base stations do not serve as data sources or data destinations. Instead, they only help relay packets for the nodes.

Besides, we consider two kinds of fairness constraints throughout this paper: throughput-fairness and bandwidthfairness. Under throughput-fairness constraint, nodes are guaranteed the same throughput, whereas under bandwidth-fairness constraint, nodes are allocated the same amount of channel bandwidth. We attempt to find out which strategy can give us higher average per-node throughput in the network. Note that these two fairness constraints are intended for infrastructure mode transmissions only. In ad hoc mode transmissions, nodes share the same bandwidth.

\section{B. Channel Capacity Model}

Let $d_{i j}$ denote the distance between a node $i$ and another node $j$. The reception power at node $j$ of the signal from node $i$, denoted by $P_{i j}$, follows the power propagation model described in [13], i.e., $P_{i j}=C P_{i} / d_{i j}^{\gamma}$, where $P_{i}$ is the transmission power of node $i, \gamma$ is the path loss exponent, and $C$ is a constant related to the antenna profiles of the transmitter and the receiver, wavelength, and so on. As a common assumption, we assume $\gamma>2$ in outdoor environments [13].

We consider the Shannon Capacity as the channel capacity between two nodes. Specifically, a transmission from node $i$ to node $j$ can have channel capacity, $R_{i j}$, which is calculated as follows:

$$
R_{i j}=B \log _{2}\left(1+S I N R_{i j}\right),
$$

where $B$ is the channel bandwidth, and $S I N R_{i j}$ is the SINR (Signal-to-Interference plus Noise Ratio) of the signal from node $i$ to node $j$. In this study, we consider the channel bandwidth $B$ to be a constant. Note that according to (1), the channel capacity can be very large if the SINR goes large. Alfano et al. [2] propose another model which upper bounds the channel capacity by a constant considering physical limitations of current transceivers. However, we do not follow their approach because we want to explore the performance of the network from information theoretic point of view, regardless of the limitations we currently have.

\section{Capacity of Regular Multihop Cellular NETWORKS}

In this section, we investigate the capacity of regular multihop cellular networks. We will first find an achievable per-node throughput under two different fairness constraints, i.e., throughput-fairness and bandwidth-fairness, respectively, when all transmissions between base stations and normal nodes are one-hop, or in infrastructure mode. Then, we attempt to find out whether carrying out one-hop transmissions only for a fraction of nodes close to base stations can improve 


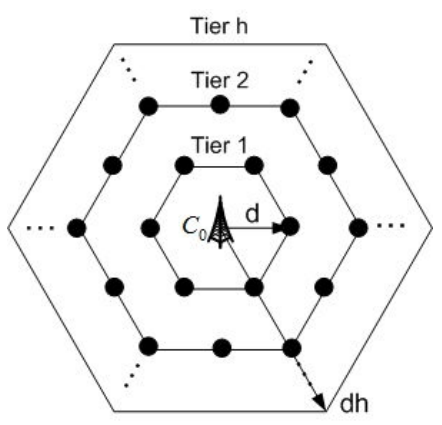

Fig. 1. An arbitrary cell in a regular multihop cellular network.

the per-node throughput. The other nodes farther away need communicate via multiple hops, i.e., in ad hoc mode, with some of the above nodes in order to communicate with base stations. We call the communications in this paradigm "hybrid mode" communications.

Recall that all packets from or to the $n$ nodes are relayed through base stations. Thus, transmissions from a source node to its destination can be carried out in three steps: Step I: from the source node to the base station in its cell; Step II: from this base station to the one in the destination node's cell; Step III: from that base station to the destination node. Base stations perform transmissions in Frequency Division Multiplexed (FDM) mode, which means that in each cell the total bandwidth $B$ is divided into many non-overlapping subchannels each of which is further divided into two equal parts for uplink and downlink transmissions, respectively. We first analyze the downlink transmissions in Step III as follows.

Consider an arbitrary cell in a regular multihop cellular network. As shown in Fig. 1, a base station $C_{0}$ is at the center of a cell, and the nodes in the cell are placed in tiers. Denote the distance between adjacent nodes by $d$, and the number of tiers in a cell by $h$, respectively. For a node at tier $q$, the reception power of a signal from the base station, denoted by $P_{r}^{q}$, satisfies $P_{r}^{q} \geq C P_{B} /(d q)^{\gamma}$, where $P_{B}$ is the signal transmission power which is the same for all base stations. The cumulative interference suffered by this node under observation, denoted by $I^{q}$, comes from all the other base stations which form outer tiers of concentric hexagons centered at $C_{0}$. We call the tiers formed by nodes in a cell inner tiers hereafter. At the $j$ th outer tier, there are at most $6 j$ interfering base stations that are at least $\frac{\sqrt{3}}{2} \cdot j \sqrt{3} d h-d h$, i.e., $\left(\frac{3}{2} j-1\right) d h$ away from the receiver node of $C_{0}$. So we can obtain

$$
\begin{aligned}
I^{q} & \leq \sum_{j=1}^{+\infty} 6 j \times \frac{C P_{B}}{\left[\left(\frac{3}{2} j-1\right) d h\right]^{\gamma}} \\
& \leq \frac{6 \cdot 2^{\gamma} C P_{B}}{(d h)^{\gamma}}\left[1+\int_{0}^{+\infty} \frac{1}{(3 j+1)^{\gamma-1}} \mathrm{~d} j\right] \\
& =\frac{6 \cdot 2^{\gamma} C P_{B}}{(d h)^{\gamma}} \cdot \frac{3 \gamma-5}{3 \gamma-6} .
\end{aligned}
$$

As a result, the SINR of the transmission from a base station to a node at inner tier $q$, denoted by $S I N R_{q}$, is as follows:

$$
S I N R_{q} \geq \frac{\gamma-2}{(3 \gamma-5) 2^{\gamma+1}} \cdot\left(\frac{h}{q}\right)^{\gamma}
$$

where noise is ignored since we consider an interference dominated environment like in [7] [14].

Note that we can easily find that uplink transmissions in Step I can have the same lower bound on SINR as shown above, which implies that uplink and downlink transmissions can have the same transmission rate, too. Since the backbone wired network has large enough bandwidth, the per-node throughput in the network is simply decided by the uplink or downlink transmissions only. In what follows, we only focus on the downlink throughput.

\section{A. Capacity in Infrastructure Mode}

We first study the capacity when all transmissions are carried out in infrastructure mode. As mentioned before, under throughput-fairness constraint, all nodes need to have the same throughput, which we denote by $\lambda^{T}(n)$. We further denote the bandwidth allocated to the downlink transmissions of each node at inner tier $q$ by $B_{q}$. Then, we have

$$
\sum_{q=1}^{h} 6 q B_{q}=B / 2 .
$$

Since $\lambda^{T}(n)=B_{q} \log _{2}\left(1+S I N R_{q}\right)$, we can get

$$
\sum_{q=1}^{h} 6 q \frac{\lambda^{T}(n)}{\log _{2}\left(1+S I N R_{q}\right)}=B / 2,
$$

and hence

$$
\lambda^{T}(n)=\frac{B / 12}{\sum_{q=1}^{h} \frac{q}{\log _{2}\left(1+S I N R_{q}\right)}} .
$$

Thus, from (2), a lower bound on the per-node throughput can be found as follows:

$$
\begin{aligned}
\lambda^{T}(n) & \geq \frac{B / 12}{\sum_{q=1}^{h} \frac{q}{\log _{2}\left(1+\frac{\gamma-2}{(3 \gamma-5) 2^{\gamma+1}}\left(\frac{h}{q}\right)^{\gamma}\right)}} \\
& \geq \frac{B / 12}{\sum_{q=1}^{h} \frac{q}{\log _{2}\left(1+\frac{\gamma-2}{(3 \gamma-5) 2^{\gamma+1}}\right)}} \\
& =\frac{m}{n} \frac{B}{2} \log _{2}\left(1+\frac{\gamma-2}{(3 \gamma-5) 2^{\gamma+1}}\right) .
\end{aligned}
$$

The last step is because of $\sum_{q=1}^{h} 6 q=n / m$. We can thus observe that when all transmissions are carried out in infrastructure mode, the per-node throughput under throughputfairness constraint is lower bounded by $m / n$, i.e., inversely proportional to the number of nodes in a cell.

We also denote the per-node throughput under bandwidthfairness constraint by $\lambda^{B}(n)$, the transmission rate of nodes at inner tier $q$ by $\lambda_{q}^{B}(n)$, and the bandwidth allocated to downlink 
transmissions of each node by $B_{i}$, respectively. Then, we can obtain

$$
\begin{aligned}
\lambda^{B}(n) & =\frac{\sum_{q=1}^{h} 6 q \lambda_{q}^{B}(n)}{n / m} \\
& =\frac{m}{n} \sum_{q=1}^{h} 6 q B_{i} \log _{2}\left(1+S I N R_{q}\right) .
\end{aligned}
$$

From (2), a lower bound on $\lambda^{B}(n)$ can be obtained by

$$
\begin{aligned}
\lambda^{B}(n) & \geq \frac{m}{n} \sum_{q=1}^{h} 6 q B_{i} \log _{2}\left(1+\frac{\gamma-2}{(3 \gamma-5) 2^{\gamma+1}}\left(\frac{h}{q}\right)^{\gamma}\right) \\
& \geq \frac{m}{n} \sum_{q=1}^{h} 6 q B_{i} \log _{2}\left(1+\frac{\gamma-2}{(3 \gamma-5) 2^{\gamma+1}}\right) \\
& =\frac{m}{n} \frac{B}{2} \log _{2}\left(1+\frac{\gamma-2}{(3 \gamma-5) 2^{\gamma+1}}\right) .
\end{aligned}
$$

The last step is due to $\sum_{q=1}^{h} 6 q B_{i}=B / 2$. We can find that when all transmissions are carried out in infrastructure mode, the per-node throughput under bandwidth-fairness constraint is also lower bounded by $m / n$, which means that sacrificing the throughput of nodes far away from base stations may not help improve the network throughput in the order sense.

\section{B. Capacity in Hybrid Mode}

Section III-A investigates the capacity when all transmissions between base stations and normal nodes are carried out in one-hop. Here, we explore whether performing transmissions in hybrid mode, i.e., some in infrastructure mode and the others in ad hoc mode, can improve network capacity.

Assume that in each cell the nodes located at tiers 1 to $g$ $(1<g<h)$ transmit packets to and receive packets from the base station directly in one hop, i.e., in infrastructure mode. For uplink transmissions, the nodes residing at tiers $g+1$ to $h$ first transmit their packets to the nodes at tier $g$ via multiple hops, i.e., in ad hoc mode, and then to the base station in infrastructure mode. Similarly, the downlink transmissions of the nodes at tiers $g+1$ to $h$ go from the base station to the nodes at tier $g$ in infrastructure mode first and then to the destination nodes in ad hoc mode. $\theta B(0<\theta<1)$ out of the total bandwidth $B$ is allocated to infrastructure mode transmissions, and the rest of the bandwidth, i.e., $(1-\theta) B$, is left for ad hoc mode transmissions. Each part is further split into two equal parts for uplink and downlink transmissions, respectively.

We first study the capacity under throughput-fairness constraint. Denote the per-node throughput in hybrid mode in the network by $\lambda_{H}^{T}(n)$, the per-node throughput in infrastructure mode by $\lambda_{H S}^{T}(n)$, and the per-node throughput in ad hoc mode by $\lambda_{H M}^{T}(n)$, respectively. Then, we get

$$
\lambda_{H}^{T}(n)=\min \left\{\lambda_{H S}^{T}(n), \lambda_{H M}^{T}(n)\right\} .
$$

Denote the bandwidth allocated to downlink transmissions of nodes at tier $q(1 \leq q \leq g)$ by $B_{q}^{\prime}$. Thus, for $1 \leq q \leq g-1$, we have

$$
\lambda_{H S}^{T}(n)=B_{q}^{\prime} \log _{2}\left(1+S I N R_{q}\right)
$$

and for $q=g$, we get

$\lambda_{H S}^{T}(n)=\frac{B_{g}^{\prime} \log _{2}\left(1+S I N R_{g}\right)}{\left(\sum_{j=g}^{h} 6 j\right) / 6 g}=\frac{B_{g}^{\prime} \log _{2}\left(1+S I N R_{g}\right)}{(h+g)(h-g+1) / 2 g}$.

Then, it follows that

$$
\begin{aligned}
& \sum_{q=1}^{g-1} \frac{6 q \lambda_{H S}^{T}(n)}{\log _{2}\left(1+S I N R_{q}\right)} \\
& \quad+\frac{6 g \lambda_{H S}^{T}(n) \cdot(h+g)(h-g+1) / 2 g}{\log _{2}\left(1+S I N R_{g}\right)}=\theta B / 2 .
\end{aligned}
$$

As a result, from (2), a lower bound on $\lambda_{H S}^{T}(n)$ is as follows:

$$
\begin{aligned}
& \lambda_{H S}^{T}(n) \geq \\
& \frac{\theta B / 12}{\left.\sum_{q=1}^{g-1} \frac{q}{\log _{2}\left(1+\frac{\gamma-2}{(3 \gamma-5) 2^{\gamma+1}}\left(\frac{h}{q}\right) \gamma\right.}+\frac{(h+g)(h-g+1) / 2}{\log _{2}\left(1+\frac{\gamma-2}{(3 \gamma-5) 2^{\gamma+1}}\left(\frac{h}{g}\right) \gamma\right.}\right)} .
\end{aligned}
$$

Let $K=\frac{\gamma-2}{(3 \gamma-5) 2^{\gamma+1}}$, and $g=\eta h$ where $1 / h<\eta<1$. We can have

$$
\begin{aligned}
\lambda_{H S}^{T}(n) & \geq \frac{\theta B / 12}{\sum_{q=1}^{g-1} \frac{q}{\log _{2}\left(1+K\left(\frac{h}{q}\right)^{\gamma}\right)}+\frac{(h+g)(h-g+1) / 2}{\log _{2}\left(1+K\left(\frac{h}{g}\right)^{\gamma}\right)}} \\
& \geq \frac{\theta B / 12}{\frac{\eta^{2} h^{2} / 2}{\log _{2}\left(1+K / \eta^{\gamma}\right)}+\frac{\left(1-\eta^{2}\right) h^{2} / 2}{\log _{2}\left(1+K / \eta^{\gamma}\right)}} \\
& \sim \frac{m}{n} \frac{\theta B}{2} \log _{2}\left(1+K / \eta^{\gamma}\right) .
\end{aligned}
$$

Let $\eta=h^{\nu}$ where $-1<\nu<0$. We can get

$$
\begin{aligned}
\lambda_{H S}^{T}(n) & \geq \frac{m}{n} \frac{\theta B}{2} \log _{2}\left(1+K h^{-\nu \gamma}\right) \\
& \geq \frac{m}{n} \frac{\theta B}{2} \log _{2}\left(K\left(\frac{n}{3 m}\right)^{-\frac{\nu \gamma}{2}}\right) \\
& \sim \frac{m}{n} \frac{(-\nu)(1-\beta) \theta \gamma B}{4} \log _{2} n .
\end{aligned}
$$

For the ad hoc mode transmissions from nodes at tier $g$ to nodes further away from the base station, assume that all transmitters employ the same transmission power $\bar{P}$ so that the transmission range is $d$, the distance between two adjacent nodes. Consider a receiver $j$ engaged in ad hoc mode transmissions. We denote the reception power at $j$ of the signal from the corresponding transmitter and of the cumulative interference to $j$ by $P_{r}^{j}$ and $I^{j}$, respectively. Then, we have

$$
P_{r}^{j}=\frac{C \bar{P}}{d^{\gamma}}
$$

and

$$
I^{j} \leq \sum_{k=1}^{+\infty} 6 k \frac{C \bar{P}}{\left(\frac{\sqrt{3}}{2} k d\right)^{\gamma}} \leq \frac{6 C \bar{P}}{\left(\frac{\sqrt{3}}{2} d\right)^{\gamma}} \zeta(\gamma-1) .
$$

So, the SINR of the transmission, denoted by $S I N R_{j}$, satisfies

$$
\operatorname{SINR}_{j} \geq \frac{1}{6 \zeta(\gamma-1)}\left(\frac{\sqrt{3}}{2}\right)^{\gamma} .
$$


Note that the transmission range is $d$. We also set the carrier sensing range to $d$ so that there are at most 7 nodes in the sensing area which share the same channel. Since nodes at tier $g$ have to relay packets for all nodes at tiers $g+1$ to $h$, a per-node throughput $\lambda_{H M}^{T}(n)$ is feasible if

$$
\lambda_{H M}^{T}(n) \cdot \bar{H} \leq \frac{(1-\theta)(B / 2) \log _{2}\left(1+\underline{S I N R_{j}}\right)}{7}
$$

where $S I N R_{j}$ is the lower bound on $S I N R_{j}, \bar{H}$ is the average ad hoc mode relay burden for each node at tier $g$, and

$$
\bar{H}=\frac{\sum_{q=g+1}^{h} 6 q}{6 g}=\frac{(g+h+1)(h-g)}{2 g} \sim \frac{1-\eta^{2}}{2 \eta} h .
$$

From (7) and (8), a lower bound on $\lambda_{H M}^{T}(n)$ can thus be calculated as:

$$
\begin{aligned}
\lambda_{H M}^{T}(n)= & \frac{\eta(1-\theta) B}{7\left(1-\eta^{2}\right) h} \log _{2}\left(1+\underline{S_{I N R_{j}}}\right) \\
= & \frac{(1-\theta) B}{7\left(1-h^{2 \nu}\right) h^{1-\nu}} \log _{2}\left(1+\underline{S_{I N R_{j}}}\right) \\
\sim & \left(\frac{3 m}{n}\right)^{\frac{1-\nu}{2}} \cdot \frac{(1-\theta) B}{7} \\
& \cdot \log _{2}\left(1+\frac{1}{6 \zeta(\gamma-1)}\left(\frac{\sqrt{3}}{2}\right)^{\gamma}\right) .
\end{aligned}
$$

Since $-1<\nu<0$, we have $\frac{1}{2}<\frac{1-\nu}{2}<1$. Substituting (6) and (9) into (5), we can find that the maximum feasible throughput $\lambda_{H}^{T}(n)$ is achieved when $\nu \rightarrow-1^{+}$and $\theta=1-$ $f \rightarrow 1^{-}$where $f$ is a function that decays exponentially slower than $\left(\frac{m}{n}\right)^{\frac{1+\nu}{2}}$, and

$$
\lambda_{H}^{T}(n) \geq \frac{m}{n} \frac{(1-\beta) \gamma B}{4} \log _{2} n .
$$

We then explore the per-node throughput in hybrid mode under bandwidth-fairness constraint, denoted by $\lambda_{H}^{B}(n)$. Specifically, the nodes at tiers 1 to $g$ equally share the total bandwidth of $\theta B$ allocated to infrastructure mode transmissions, and the other nodes at tiers $g$ to $h$ share the $(1-\theta) B$ bandwidth left for ad hoc mode transmissions. Recall that nodes at tier $g$ act as relay nodes to/from base stations for the nodes at tiers $g+1$ to $h$. We also assume that nodes at tiers $g$ to $h$ have the same throughput. We can have $B_{i}^{\prime} \cdot \sum_{q=1}^{g} 6 q=\theta B / 2$, where $B_{i}^{\prime}$ is the downlink bandwidth for each node at tiers 1 to $g$, i.e., $B_{i}^{\prime}=\frac{\theta B}{6 g(g+1)}$.

Denote the transmission rate of a node at tier $q(1 \leq q \leq g)$ by $\lambda_{H S}^{q}(n)$, and the transmission rate of a node at tier $j(g<$ $j \leq h)$ by $\lambda_{H M}^{j}(n)$, respectively. Then, according to the lower bound on SINR obtained in (2), we can have for $1 \leq q \leq g$,

$$
\lambda_{H S}^{q}(n) \geq \frac{\theta B}{6 g(g+1)} \log _{2}\left(1+K\left(\frac{h}{q}\right)^{\gamma}\right) .
$$

Note that $6 g \lambda_{H S}^{g}(n) / \sum_{q=g}^{h} 6 q$ is the average per-node throughput for the nodes located at tiers $g$ to $h$ that can be supported by infrastructure mode transmissions at tier $g$. Since from (9) we get $\lambda_{H M}^{j}(n)=\Omega\left(\left(\frac{m}{n}\right)^{\frac{1-\nu}{2}}\right)=\omega\left(\frac{m}{n}\right)$ while the maximum of the achievable average throughput
$6 g \lambda_{H S}^{g}(n) / \sum_{q=g}^{h} 6 q$ is on the exponential order of $m / n$, i.e., $o\left(\lambda_{H M}^{j}(n)\right)$, the infrastructure mode transmissions are the bottleneck when $\theta=1-f$ where $f$ is a function that decays exponentially slower than $\left(\frac{m}{n}\right)^{\frac{1+\nu}{2}}$.

As a result, the maximum feasible per-node throughput $\lambda_{H}^{B}(n)$ can be calculated as follows:

$$
\begin{aligned}
\lambda_{H}^{B}(n) & \geq \frac{m}{n} \sum_{q=1}^{g} 6 q \cdot \frac{\theta B}{6 g(g+1)} \log _{2}\left(1+K\left(\frac{h}{q}\right)^{\gamma}\right) \\
& \geq \frac{m}{n} \frac{\theta B}{6 g(g+1)} \log _{2}\left(1+K\left(\frac{h}{g}\right)^{\gamma}\right) \cdot 3 g(g+1) \\
& =\frac{m}{n} \frac{\theta B}{2} \log _{2}\left(1+K h^{-\nu \gamma}\right) .
\end{aligned}
$$

By letting $\theta \rightarrow 1^{-}$and $\nu \rightarrow-1^{+}$, we can get

$$
\begin{aligned}
\lambda_{H}^{B}(n) & \geq \frac{m}{n} \frac{\theta B}{2} \log _{2}\left(1+K h^{-\nu \gamma}\right) \\
& \sim \frac{m}{n} \frac{(1-\beta) \gamma B}{4} \log _{2} n .
\end{aligned}
$$

According to (10) and (11), we can see that by carrying out hybrid mode transmissions in each cell, the per-node throughput can be improved by a scaling factor of $\log _{2} n$ compared to that with infrastructure mode transmissions only. We also notice that throughput-fairness and bandwidth-fairness constraints can lead to the same hybrid mode feasible throughput in regular networks.

\section{Capacity of Heterogeneous Multihop Cellular Networks}

Next, we continue to investigate the capacity of heterogeneous multihop cellular networks, where the distribution of the $n$ nodes follows a general IPP, and the $m$ base stations are uniformly and randomly placed.

Recall the definition of Voronoi Tessellation: given a set of $m$ points in a plane, Voronoi tessellation divides the domain into a set of polygonal regions, the boundaries of which are the perpendicular bisectors of the lines joining the points. From [6], we know that for every $\varepsilon>0$, there is a Voronoi tessellation with the property that every Voronoi cell contains a disk of radius $\varepsilon$ and is contained in a disk of radius $2 \varepsilon$. Then, for the $m$ base stations in a network with area $L^{2}$, we can construct a Voronoi tessellation $V_{m}$ for which

- (V1) Every Voronoi cell contains a disk of area $100 L^{2} \ln m / m$.

- (V2) Every Voronoi cell is contained in a disk of radius $2 \rho(m)$, where $\rho(m):=$ the radius of a disk of area $100 L^{2} \ln m / m$.

In this case, we consider each Voronoi cell as a cell in the network.

Consider a cell $\mathcal{A}_{j}$ in which the base station is located at $b_{j}$. Denote the area of the cell by $\left|\mathcal{A}_{j}\right|$, and the minimum and the maximum of the area by $\underline{\left|\mathcal{A}_{j}\right|}$ and $\overline{\left|\mathcal{A}_{j}\right|}$, respectively. Then, we have

$$
\underline{\left|\mathcal{A}_{j}\right|}=\pi \rho^{2}(m), \quad \overline{\left|\mathcal{A}_{j}\right|}=4 \pi \rho^{2}(m), \forall j,
$$




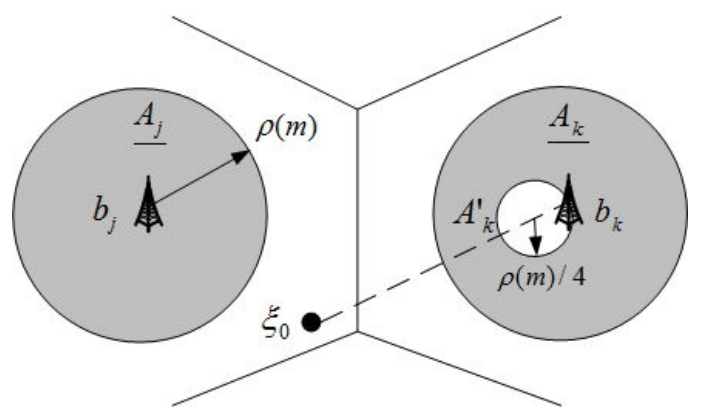

Fig. 2. Disjoint disks in a Voronoi tessellation.

and the distance from any base station to its cell boundary is at least $\rho(\mathrm{m})$ and at most $2 \rho(\mathrm{m})$. For an arbitrary point $\xi_{0} \in \mathcal{A}_{j}$, the reception power of the signal from the base station, denoted by $P_{r}\left(\xi_{0}\right)$, is

$$
P_{r}\left(\xi_{0}\right)=\frac{C P}{\left\|\xi_{0}-b_{j}\right\| \gamma}
$$

where $P$ is the signal transmission power that is the same for all base stations.

As shown in Fig. 2, since disks centered at base stations with area $\left|\mathcal{A}_{k}\right|$ are disjoint, the inside disks $\mathcal{A}_{k}^{\prime}$ with area $\left|\mathcal{A}_{k}\right| / 16$ and base stations on the disk boundaries are also disjoint. For $\xi \in \mathcal{A}_{k}^{\prime}$, we have $\left\|\xi-\xi_{0}\right\|>\rho(m) / 2$. Thus, the cumulative interference suffered by an infrastructure mode downlink reception at $\xi_{0}$, denoted by $I\left(\xi_{0}\right)$, satisfies

$$
\begin{aligned}
I\left(\xi_{0}\right) & =\sum_{k \neq j} \frac{C P}{\| \xi_{0}-b_{k}||^{\gamma}} \\
& =C P \sum_{k \neq j} \frac{1}{\left|\mathcal{A}_{k}\right| / 16} \cdot \frac{1}{\| \xi_{0}-b_{k}|| \gamma} \cdot\left(\underline{\left.\left|\mathcal{A}_{k}\right| / 16\right)}\right. \\
& \leq \frac{16 C P}{\left|\mathcal{A}_{k}\right|} \int_{\mathcal{A} \backslash\left(\mathcal{A}_{j} / 4\right)} \frac{1}{\left\|\xi_{0}-\xi\right\| \gamma} \mathrm{d} \xi \\
& =\frac{16 C P}{\left|\mathcal{A}_{j}\right|} \int_{0}^{2 \pi} \int_{\rho(m) / 2}^{+\infty} \frac{1}{l \gamma} \cdot l \mathrm{~d} l \mathrm{~d} \phi \\
& =\frac{2^{\gamma+3} C P}{\gamma-2}[\rho(m)]^{-\gamma} .
\end{aligned}
$$

Thus, the SINR of the reception at point $\xi_{0}$, denoted by $\operatorname{SINR}\left(\xi_{0}\right)$, can be calculated as follows:

$$
\operatorname{SINR}\left(\xi_{0}\right) \geq \frac{C P /\left\|\xi_{0}-b_{j}\right\|^{\gamma}}{\frac{2^{\gamma+3} C P}{\gamma-2} /[\rho(m)]^{\gamma}}=\frac{\gamma-2}{2^{\gamma+3}} \frac{\rho^{\gamma}(m)}{\left\|\xi_{0}-b_{j}\right\|^{\gamma}} .
$$

\section{A. Capacity under Throughput-fairness Constraint}

Consider a cell $\mathcal{A}_{j}$. We denote the per-node throughput under throughput-fairness constraint when all transmissions are carried out in infrastructure mode by $\widetilde{\lambda}^{T}(n)$, and the bandwidth allocated to downlink transmissions at point $\xi_{0} \in \mathcal{A}_{j}$ by $B\left(\xi_{0}\right)$. Thus, we have

$$
\widetilde{\lambda}^{T}(n)=B\left(\xi_{0}\right) \log _{2}\left(1+\operatorname{SINR}\left(\xi_{0}\right)\right),
$$

and hence

$$
\int_{\mathcal{A}_{j}} \Psi\left(\xi_{0}\right) \frac{\widetilde{\lambda}^{T}(n)}{\log _{2}\left(1+\operatorname{SINR}\left(\xi_{0}\right)\right)} \mathrm{d} \xi_{0}=B / 2 .
$$

For $\xi \in \mathcal{A}_{j}$, we get $\left\|\xi-b_{j}\right\| \leq 2 \rho(m)$. So from (13), we know that

$$
\operatorname{SINR}(\xi) \geq \frac{\gamma-2}{2^{\gamma+3}} \frac{\rho^{\gamma}(m)}{\left\|\xi-b_{j}\right\|^{\gamma}} \geq \frac{\gamma-2}{2^{2 \gamma+3}} .
$$

As a result, a lower bound on the per-node throughput can be found as follows:

$$
\begin{aligned}
\widetilde{\lambda}^{T}(n) & =\frac{B / 2}{\int_{\mathcal{A}_{j}} \Psi\left(\xi_{0}\right) \frac{1}{\log _{2}\left(1+\operatorname{SINR}\left(\xi_{0}\right)\right)} \mathrm{d} \xi_{0}} \\
& \geq \frac{B / 2}{\bar{\Psi} \cdot \frac{1}{\log _{2}\left(1+\frac{\gamma-2}{2^{2 \gamma+3}}\right)} \cdot \overline{\left|\mathcal{A}_{j}\right|}} \\
& =\frac{B}{8 \pi \bar{\Psi} \rho^{2}(m)} \log _{2}\left(1+\frac{\gamma-2}{2^{2 \gamma+3}}\right) \\
& =\frac{n^{\beta-2 \alpha}}{\bar{\Psi} \ln n} \cdot \frac{B}{800 \beta} \log _{2}\left(1+\frac{\gamma-2}{2^{2 \gamma+3}}\right) .
\end{aligned}
$$

Note that we have $n^{\beta}=o(n)$ and $n \leq \bar{\Psi} n^{2 \alpha}$. Thus, we can get $\bar{\Psi} n^{2 \alpha-\beta}=\omega(1)$, which leads to $\widetilde{\lambda}^{T}(n)=o(1 / \ln n)$.

We then study the capacity when transmissions are carried out in hybrid mode. We denote the per-node throughput in hybrid mode by $\widetilde{\lambda}_{H}^{T}(n)$, the per-node throughput in infrastructure mode by $\widetilde{\lambda}_{H S}^{T}(n)$, and the per-node throughput in ad hoc mode by $\widetilde{\lambda}_{H M}^{T}(n)$, respectively.

As shown in Fig. 3 , in a cell $\mathcal{A}_{j}$, the nodes in areas $S_{1}$ and $S_{2}$ carry out transmissions in infrastructure mode, while the nodes in area $S_{3}$ perform transmissions in ad hoc mode, for which the nodes in $S_{2}$ act as relay nodes from or to the base station. Let $\varepsilon$ be the ratio of the number of nodes in areas $S_{2}$ and $S_{3}$ to that in area $S_{2}$, i.e.,

$$
\varepsilon=\frac{\int_{S_{2} \cup S_{3}} \Psi(\xi) \mathrm{d} \xi}{\int_{S_{2}} \Psi(\xi) \mathrm{d} \xi} \leq \frac{\bar{\Psi}\left(\left|S_{2}\right|+\left|S_{3}\right|\right)}{\underline{\Psi}\left|S_{2}\right|} .
$$

Then, we have

$$
\begin{aligned}
& \int_{S_{1}} \Psi\left(\xi_{0}\right) \frac{\widetilde{\lambda}_{H S}^{T}(n)}{\log _{2}\left(1+S I N R\left(\xi_{0}\right)\right)} \mathrm{d} \xi_{0} \\
& \quad+\int_{S_{2}} \Psi\left(\xi_{0}\right) \frac{\varepsilon \widetilde{\lambda}_{H S}^{T}(n)}{\log _{2}\left(1+\operatorname{SINR}\left(\xi_{0}\right)\right)} \mathrm{d} \xi_{0}=\theta B / 2,
\end{aligned}
$$

from which we can obtain

$$
\theta B / 2 \leq \bar{\Psi} \frac{\widetilde{\lambda}_{H S}^{T}(n)\left|S_{1}\right|}{\log _{2}(1+\underline{S I N R})}+\bar{\Psi} \frac{\bar{\varepsilon} \widetilde{\lambda}_{H S}^{T}(n)\left|S_{2}\right|}{\log _{2}(1+\underline{S I N R})},
$$

where $\underline{S I N R}$ and $\bar{\varepsilon}$ are the minimum of SINR and the maximum of $\varepsilon$ derived in (13) and (15), respectively. Thus, we have

$$
\begin{aligned}
\widetilde{\lambda}_{H S}^{T}(n) & \geq \frac{(\theta B / 2) \log _{2}(1+\underline{S I N R})}{\bar{\Psi}\left|S_{1}\right|+\bar{\varepsilon}\left|S_{2}\right|} \\
& =\frac{(\theta B / 2) \underline{\Psi} \log _{2}(1+\underline{S I N R})}{\underline{\Psi} \bar{\Psi}\left|S_{1}\right|+\bar{\Psi}^{2}\left(\left|S_{2}\right|+\left|S_{3}\right|\right)} \\
& \geq \frac{(\theta B / 2) \underline{\Psi} \log _{2}(1+\underline{S I N R})}{\bar{\Psi}^{2}\left(\left|S_{1}\right|+\left|S_{2}\right|+\left|S_{3}\right|\right)} \\
& \geq \frac{\theta B \underline{\Psi}}{8 \pi \bar{\Psi}^{2} \rho^{2}(m)} \log _{2}(1+\underline{S I N R}) .
\end{aligned}
$$




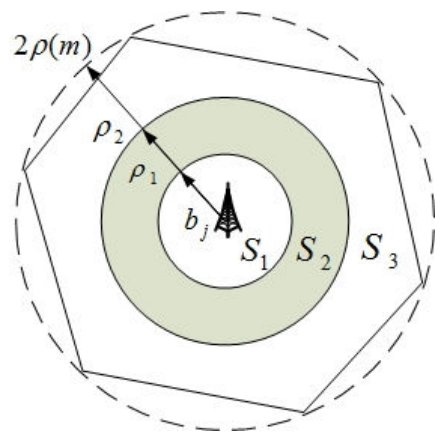

Fig. 3. A Voronoi cell $\mathcal{A}_{j}$ in a heterogeneous multihop cellular network with $\mathcal{A}_{j}=S_{1} \cup S_{2} \cup S_{3}$.

For ad hoc mode transmissions, we choose the transmission power $\widetilde{P}$ such that the transmission area $\left|S_{t}\right|=c_{g} \ln n / \underline{\Psi}$ where $c_{g}>6 /(4 \pi-3 \sqrt{3})$. Note that since $\underline{\Psi} n^{2 \alpha}=\omega(\ln n)$, we have $\left|S_{t}\right|=o\left(n^{2 \alpha}\right)$ and hence $S_{t} \subset \mathcal{A}$. Then, from [16], we know that under this condition any node can find a local neighbor closer to the destination than itself, and hence the multi-hop transmissions from/to the nodes in $S_{2}$ can always be successful.

Consider a receiver $j$ located at point $\xi_{0} \in S_{3}$ engaged in ad hoc mode transmissions. We denote the reception power of the signal from the corresponding transmitter and of the cumulative interference to the receiver by $\widetilde{P}_{r}\left(\xi_{0}\right)$ and $\widetilde{I}\left(\xi_{0}\right)$, respectively. Then, we have

$$
\widetilde{P}_{r}\left(\xi_{0}\right) \geq \frac{C \widetilde{P}}{\left(\sqrt{\left|S_{t}\right| / \pi}\right)^{\gamma}} .
$$

We also set the carrier sensing range to be twice the transmission range. Note that within the same carrier sensing range, only one node can transmit. Let $\mathbb{T}$ denote the set of all nodes transmitting when receiver $j$ is receiving. Then, disks centered at transmitters in $\mathbb{T}$ with area $\left|S_{t}\right|$ are disjoint, and so are the inside disks with area $\left|S_{t}\right| / 16$ like in Fig. 2. Similar to (12), we can get

$$
\widetilde{I}\left(\xi_{0}\right) \leq \frac{2^{\gamma+3} C \widetilde{P}}{\gamma-2} \frac{1}{\left(\sqrt{\left|S_{t}\right| / \pi}\right)^{\gamma}} .
$$

Thus, the SINR of the reception at point $\xi_{0}$, denoted by $\widehat{\operatorname{SINR}}\left(\xi_{0}\right)$, is as follows:

$$
\widetilde{S I N R}\left(\xi_{0}\right) \geq \frac{C \widetilde{P} /\left(\sqrt{\left|S_{t}\right| / \pi}\right)^{\gamma}}{\frac{2 \gamma+3 C \widetilde{P}}{\gamma-2} /\left(\sqrt{\left|S_{t}\right| / \pi}\right)^{\gamma}}=\frac{\gamma-2}{2^{\gamma+3}},
$$

which can be lower bounded by a constant.

Referring to Fig. 3, denote the area where $\rho_{2} \leq\left\|\xi-b_{j}\right\| \leq$ $2 \rho(m)$ by $\widehat{S}_{3}$. Then, $S_{3} \subset \widehat{S}_{3}$. Besides, we also set $\rho_{2}-\rho_{1}=$ $\sqrt{\left|S_{t}\right| / \pi}$. Thus, the average ad hoc mode relay burden for each node in $S_{2}$, denoted by $\widetilde{H}$, can be calculated as follows:

$$
\widetilde{H} \leq \frac{\int_{\widehat{S}_{3}} \Psi(\xi) \mathrm{d} \xi}{\int_{S_{2}} \Psi(\xi) \mathrm{d} \xi} \leq \frac{\bar{\Psi}\left|\widehat{S}_{3}\right|}{\underline{\Psi}\left|S_{2}\right|} .
$$

Note that in the carrier sensing area, there are at most $4 \bar{\Psi}\left|S_{t}\right|$ nodes which share the same channel. Thus, a per-node throughput $\widetilde{\lambda}_{H M}^{T}(n)$ is feasible if

$$
\widetilde{\lambda}_{H M}^{T}(n) \cdot \widetilde{H} \leq \frac{(1-\theta)(B / 2) \log _{2}\left(1+(\gamma-2) / 2^{\gamma+3}\right)}{4 \bar{\Psi}\left|S_{t}\right|} .
$$

So, from (17), a lower bound on $\widetilde{\lambda}_{H M}^{T}(n)$ can be found by

$$
\begin{aligned}
\widetilde{\lambda}_{H M}^{T}(n) & =\frac{(1-\theta)(B / 2)}{4 \bar{\Psi} \widetilde{H}\left|S_{t}\right|} \log _{2}\left(1+\frac{\gamma-2}{2^{\gamma+3}}\right) \\
& =\frac{\underline{\Psi}\left|S_{2}\right|}{\bar{\Psi}^{2}\left|S_{t}\right| \cdot\left|\widehat{S}_{3}\right|} \frac{(1-\theta) B}{8} \log _{2}\left(1+\frac{\gamma-2}{2^{\gamma+3}}\right) \\
& \geq \frac{\underline{\Psi}\left|S_{2}\right|}{\bar{\Psi}^{2} \rho^{2}(m)\left|S_{t}\right|} \frac{(1-\theta) B}{32 \pi} \log _{2}\left(1+\frac{\gamma-2}{2^{\gamma+3}}\right)
\end{aligned}
$$

Again, note that the per-node throughput in the network can be obtained by:

$$
\widetilde{\lambda}_{H}^{T}(n)=\min \left\{\widetilde{\lambda}_{H S}^{T}(n), \widetilde{\lambda}_{H M}^{T}(n)\right\} .
$$

Comparing the results in (16) and (18), we can have the following results.

Case I: $\rho(m)=\omega(\sqrt{\ln n / \underline{\Psi}})$, i.e., $\underline{\Psi}=\omega\left(n^{\beta-2 \alpha}\right)$. In this case, $\rho(m)=\omega\left(\sqrt{\left|S_{t}\right|}\right)$, and $\bar{\Psi}=\Omega(\underline{\Psi})=\omega\left(n^{\beta-2 \alpha}\right)$.

i) When $\rho(m)=\omega(1)$, let $\rho(m)=\rho^{\sigma}(m) \sqrt{\left|S_{t}\right| / \pi}$ where $\sigma>0$. We choose $\left|S_{2}\right|=\rho^{\tau}(m)\left|S_{t}\right|$ where $0<\tau<\sigma$. Then, since $\pi \rho_{2}^{2}-\pi\left(\rho_{2}-\sqrt{\left|S_{t}\right| / \pi}\right)^{2}=\left|S_{2}\right|$, we can have that $\rho_{2} \sim$ $\rho^{\tau}(m) \sqrt{\left|S_{t}\right| / \pi} / 2$. As a result, $\pi \rho^{2}(m)=\rho^{2 \sigma}\left|S_{t}\right|=\omega\left(\pi \rho_{2}^{2}\right)$. Since $\pi \rho^{2}(m)<\left|\mathcal{A}_{j}\right|$, we have $S_{2} \subset \mathcal{A}_{j}$, i.e., the chosen $S_{2}$ is totally contained by the cell area $\mathcal{A}_{j}$. Besides, it is also easy to show that in $S_{2}$ there are always some nodes, i.e., $S_{2}$ is not empty. So we can see that (18) changes into

$\tilde{\lambda}_{H M}^{T}(n) \geq \frac{\underline{\Psi}}{\bar{\Psi}^{2} \rho^{2-\tau}(m)} \cdot \frac{(1-\theta) B}{32 \pi} \log _{2}\left(1+\frac{\gamma-2}{2^{\gamma+3}}\right)$.

Recall that in (16), $\underline{S I N R}=\frac{\gamma-2}{8} \frac{\rho^{\gamma}(m)}{\left\|\xi_{0}-b_{j}\right\|^{\gamma}}$ where $\xi_{o} \in S_{1} \cup$ $S_{2}$. Therefore we can have

$$
\begin{aligned}
\underline{\operatorname{SINR}} & \geq \frac{\gamma-2}{2^{\gamma+3}}\left(\frac{\rho(m)}{\rho^{\tau}(m) \sqrt{\left|S_{t}\right| / \pi} / 2}\right)^{\gamma} \\
& =c_{0}[\rho(m)]^{(\sigma-\tau) \gamma}
\end{aligned}
$$

where $c_{0}=(\gamma-2) / 8$. Thus, the feasible throughput in infrastructure mode $\widetilde{\lambda}_{H S}^{T}(n)$ is as follows:

$$
\tilde{\lambda}_{H S}^{T}(n) \geq \frac{\theta B \underline{\Psi}}{8 \pi \bar{\Psi}^{2} \rho^{2}(m)} \log _{2}\left(1+c_{0}[\rho(m)]^{(\sigma-\tau) \gamma}\right) .
$$

Since $0<\tau<\sigma$ and $\rho(m)=\omega(1)$, we can see that infrastructure mode transmissions are the bottleneck as $\theta=1-\widetilde{f}$ where $\widetilde{f}$ decays exponentially slower than $\rho^{-\tau}(m)$, and hence the feasible throughput in hybrid mode $\widetilde{\lambda}_{H}^{T}(n)$ is as follows:

$$
\begin{aligned}
\widetilde{\lambda}_{H}^{T}(n) & =\frac{B \underline{\Psi}}{8 \pi \bar{\Psi}^{2} \rho^{2}(m)} \log _{2}\left(1+c_{0}\left(\frac{100 L^{2} \ln m}{\pi m}\right)^{\frac{(\sigma-\tau) \gamma}{2}}\right) \\
& \sim \frac{c_{t} B \underline{\Psi}}{8 \pi \bar{\Psi}^{2} \rho^{2}(m)} \log _{2} n \\
& =\frac{\Psi n^{\beta-2 \alpha}}{\bar{\Psi}^{2}} \cdot \frac{c_{t} B}{800 \beta \ln 2}
\end{aligned}
$$


where $c_{t}=(2 \alpha-\beta)(\sigma-\tau) \gamma / 2>0$. The maximum of $\widetilde{\lambda}_{H}^{T}(n)$ is achieved when $\theta \rightarrow 1^{-}$and $\tau \rightarrow 0^{+}$.

ii) When $\rho(m)=o(1)$, let $\rho(m)=\rho^{-\sigma}(m) \sqrt{\left|S_{t}\right| / \pi}$ where $\sigma>0$. We choose $\left|S_{2}\right|=\rho^{-\tau}(m)\left|S_{t}\right|$ where $0<\tau<\sigma$. Then, similar to i), we can find that the feasible throughput in hybrid mode $\widetilde{\lambda}_{H}^{T}(n)$ is as follows:

$$
\begin{aligned}
\widetilde{\lambda}_{H}^{T}(n) & \sim \frac{c_{t}^{\prime} B \underline{\Psi}}{8 \pi \bar{\Psi}^{2} \rho^{2}(m)} \log _{2} n \\
& =\frac{\underline{\Psi} n^{\beta-2 \alpha}}{\bar{\Psi}^{2}} \cdot \frac{c_{t}^{\prime} B}{800 \beta \ln 2}
\end{aligned}
$$

where $c_{t}^{\prime}=(2 \alpha-\beta)(\sigma-\tau) \gamma / 2>0$. The maximum of $\widetilde{\lambda}_{H}^{T}(n)$ is achieved when $\theta \rightarrow 1^{-}$and $\tau \rightarrow 0^{+}$.

iii) When $\rho(m)=\Theta(1)$, since $\rho(m)=\omega\left(\sqrt{\left|S_{t}\right|}\right)$, we have $\left|S_{t}\right|=o(1)$. We choose $\left|S_{2}\right|=\left|S_{t}\right|^{\tau}$ where $1 / 2<\tau<1$. Similarly, we can have $\rho_{2} \sim\left|S_{t}\right|^{\tau-\frac{1}{2}} /(2 \sqrt{\pi})$, and $\pi \rho_{2}^{2}=$ $o\left(\pi \rho^{2}(m)\right)$. Thus, we can make sure that $S_{2}$ is not empty since $\left|S_{2}\right|=\omega\left(\left|S_{t}\right|\right)$ and $S_{t}$ is not empty, and that $S_{2} \subset \mathcal{A}_{j}$. Thus, (18) changes into

$\widetilde{\lambda}_{H M}^{T}(n) \geq \frac{\underline{\Psi}}{\bar{\Psi}^{2} \rho^{2}(m)\left|S_{t}\right|^{1-\tau}} \cdot \frac{(1-\theta) B}{32 \pi} \log _{2}\left(1+\frac{\gamma-2}{2^{\gamma+3}}\right)$.

For infrastructure mode transmissions, we can get

$$
\begin{aligned}
\underline{S I N R} & =\frac{\gamma-2}{2^{\gamma+3}}\left(\frac{\rho(m)}{\left|S_{t}\right|^{\tau-\frac{1}{2}} /(2 \sqrt{\pi})}\right)^{\gamma} \\
& =c_{0}(\sqrt{\pi} \rho(m))^{\gamma}\left|S_{t}\right|^{\left(\frac{1}{2}-\tau\right) \gamma},
\end{aligned}
$$

and hence the feasible throughput $\widetilde{\lambda}_{H S}^{T}(n)$ is as follows:

$\widetilde{\lambda}_{H S}^{T}(n) \geq \frac{\theta B \underline{\Psi}}{8 \pi \bar{\Psi}^{2} \rho^{2}(m)} \log _{2}\left(1+c_{0}(\sqrt{\pi} \rho(m))^{\gamma}\left|S_{t}\right|^{\left(\frac{1}{2}-\tau\right) \gamma}\right)$.

Since $1 / 2<\tau<1$ and $\left|S_{t}\right|=o(1)$, we can find that infrastructure mode transmissions are the bottleneck as $\theta=1-\tilde{f}$ where $\widetilde{f}$ decays exponentially slower than $\left|S_{t}\right|^{1-\tau}$. By letting $\left|S_{t}\right|=n^{-\kappa}$ where $\kappa>0$, the feasible throughput in hybrid mode $\widetilde{\lambda}_{H}^{T}(n)$ can be found by

$$
\begin{aligned}
\widetilde{\lambda}_{H}^{T}(n) & =\frac{B \underline{\Psi}}{8 \pi \bar{\Psi}^{2} \rho^{2}(m)} \log _{2}\left(1+c_{0}(\sqrt{\pi} \rho(m))^{\gamma} n^{\left(\tau-\frac{1}{2}\right) \kappa \gamma}\right) \\
& \sim \frac{c_{t}^{\prime \prime} B \underline{\Psi}}{8 \pi \bar{\Psi}^{2} \rho^{2}(m)} \log _{2} n \\
& =\frac{\underline{\Psi} n^{\beta-2 \alpha}}{\bar{\Psi}^{2}} \cdot \frac{c_{t}^{\prime \prime} B}{800 \beta \ln 2}
\end{aligned}
$$

where $c_{t}^{\prime \prime}=\left(\tau-\frac{1}{2}\right) \kappa \gamma>0$. The maximum of $\widetilde{\lambda}_{H}^{T}(n)$ is achieved when $\theta \rightarrow 1^{-}$and $\tau \rightarrow 1^{-}$.

Comparing (14) with (19) to (21), we can see that in Case I, when $\bar{\Psi} / \underline{\Psi}=o\left(\log _{2} n\right)$, hybrid mode transmissions can yield higher throughput than pure infrastructure mode transmissions, and the throughput gain can be on the order of $\log _{2} n$ when $\underline{\Psi}=\Theta(\bar{\Psi})$.

Case II: $\rho(m)=\mathrm{O}(\sqrt{\ln n / \underline{\Psi}})$, i.e., $\underline{\Psi}=\mathrm{O}\left(n^{\beta-2 \alpha}\right)$. In this case, $\rho(m)=\mathrm{O}\left(\sqrt{\left|S_{t}\right|}\right)$, and thus $\left|S_{2}\right|<4 \pi \rho^{2}(m)=\mathrm{O}\left(\left|S_{t}\right|\right)$.
Comparing (16) with (18), we can see that ad hoc mode transmissions are the bottleneck as $\theta$ decays slower than $\left|S_{2}\right| /\left|S_{t}\right|$. The maximum feasible per-node throughput in hybrid mode is then achieved when $\left|S_{1}\right|=0,\left|S_{2}\right|=\pi \rho^{2}(m)$, and $\theta$ decays exponentially slower than $\underline{\Psi} n^{2 \alpha-\beta}$, i.e.,

$$
\widetilde{\lambda}_{H}^{T}(n)=\frac{\underline{\Psi} B}{32 \bar{\Psi}^{2}\left|S_{t}\right|} \log _{2}\left(1+\frac{\gamma-2}{2^{\gamma+3}}\right) .
$$

Comparing (14) with (22), we get

$$
\frac{\widetilde{\lambda}_{H}^{T}(n)}{\widetilde{\lambda}^{T}(n)}=\Theta\left(\frac{\underline{\bar{\Psi}}}{\bar{\Psi}} \cdot \frac{\rho^{2}(m)}{\left|S_{t}\right|}\right)=\mathrm{O}(1),
$$

which means that when the minimum node density is smaller than the density of base stations in the network, hybrid mode transmissions will not help and we should carry out all transmissions in infrastructure mode.

\section{B. Capacity under Bandwidth-fairness Constraint}

We first consider the case when all transmissions in each cell are performed in infrastructure mode. Denote the per-node throughput under bandwidth-fairness constraint by $\widetilde{\lambda}^{B}(n)$, and the bandwidth allocated to the downlink transmissions of each node by $\widetilde{B}_{i}$, respectively. Then, for a cell $\mathcal{A}_{j}$, we can have

$$
\widetilde{\lambda}^{B}(n)=\frac{\int_{\mathcal{A}_{j}} \Psi(\xi) \widetilde{B}_{i} \log _{2}(1+S I N R(\xi)) \mathrm{d} \xi}{\int_{\mathcal{A}_{j}} \Psi(\xi) \mathrm{d} \xi},
$$

and hence

$$
\begin{aligned}
\widetilde{\lambda}^{B}(n) & \geq \frac{\int_{\mathcal{A}_{j}} \Psi(\xi) \widetilde{B}_{i} \mathrm{~d} \xi}{\int_{\mathcal{A}_{j}} \Psi(\xi) \mathrm{d} \xi} \log _{2}\left(1+\frac{\gamma-2}{2^{2 \gamma+3}}\right) \\
& \geq \frac{B / 2}{\bar{\Psi} \cdot \overline{\left|\mathcal{A}_{j}\right|}} \log _{2}\left(1+\frac{\gamma-2}{2^{2 \gamma+3}}\right) \\
& =\frac{B}{8 \pi \bar{\Psi} \rho^{2}(m)} \log _{2}\left(1+\frac{\gamma-2}{2^{2 \gamma+3}}\right) \\
& =\frac{n^{\beta-2 \alpha}}{\bar{\Psi} \ln n} \cdot \frac{B}{800 \beta} \log _{2}\left(1+\frac{\gamma-2}{2^{2 \gamma+3}}\right)
\end{aligned}
$$

which is the same result as that shown in (14).

We then study the per-node throughput when transmissions in each cell are carried out in hybrid mode, which is denoted by $\widetilde{\lambda}_{H}^{B}(n)$. Consider a cell $\mathcal{A}_{j}$ as shown in Fig. 3 . Assume nodes in areas $S_{1}$ and $S_{2}$ equally share the bandwidth $\theta B$ allocated to infrastructure mode transmissions, and nodes in $S_{3}$ and in $S_{2}$ as well share the bandwidth $(1-\theta) B$ left for ad hoc mode transmissions.

Denote by $\widetilde{B}_{i}$ the downlink bandwidth for each node in areas $S_{1}$ and $S_{2}$, and by $\widetilde{\lambda}_{H S}^{B}(n)$ the per-node throughput for nodes in areas $S_{2}$ and $S_{3}$ supported by infrastructure mode transmissions in area $S_{2}$. Then, we can have

$$
\int_{S_{1} \cup S_{2}} \Psi(\xi) \widetilde{B}_{i} \mathrm{~d} \xi=\theta B / 2
$$

and hence

$$
\widetilde{B}_{i} \geq \frac{\theta B}{2 \bar{\Psi}\left(\left|S_{1}\right|+\left|S_{2}\right|\right)}
$$


Besides, we can obtain that

$$
\begin{aligned}
\widetilde{\lambda}_{H S}^{B}(n) & =\frac{\int_{S_{2}} \Psi(\xi) \widetilde{B}_{i} \log _{2}(1+S I N R(\xi)) \mathrm{d} \xi}{\int_{S_{2} \cup S_{3}} \Psi(\xi) \mathrm{d} \xi} \\
& \geq \frac{\underline{\Psi}\left|S_{2}\right| \frac{\theta B}{2 \bar{\Psi}\left(\left|S_{1}\right|+\left|S_{2}\right|\right)} \log _{2}\left(1+\frac{\gamma-2}{\left.2^{\gamma+3}\left(\frac{\rho(m)}{\rho_{2}}\right)^{\gamma}\right)}\right.}{\bar{\Psi}\left(\left|S_{2}\right|+\left|S_{3}\right|\right)} \\
& \geq \frac{\theta B \underline{\Psi}}{8 \pi \rho^{2}(m) \bar{\Psi}^{2}} \frac{\left|S_{2}\right|}{\left|S_{1}\right|+\left|S_{2}\right|} \log _{2}\left(1+\frac{\gamma-2}{2^{\gamma+3}}\left(\frac{\rho(m)}{\rho_{2}}\right)^{\gamma}\right) .
\end{aligned}
$$

Note that $\left|S_{1}\right|+\left|S_{2}\right|=\pi \rho_{2}^{2}$, which gives us

$$
\widetilde{\lambda}_{H S}^{B}(n) \geq \frac{\theta B \underline{\Psi}\left|S_{2}\right|}{8 \pi^{2} \rho^{2}(m) \bar{\Psi}^{2} \rho_{2}^{2}} \log _{2}\left(1+\frac{\gamma-2}{2^{\gamma+3}}\left(\frac{\rho(m)}{\rho_{2}}\right)^{\gamma}\right) \text {. }
$$

Recall that the ad hoc mode per-node throughput of the nodes in area $S_{3}$ is shown in (18). Comparing (18) with (24), we can have the following results.

Case I: $\rho(m)=\omega(\sqrt{\ln n / \underline{\Psi}})$, i.e., $\underline{\Psi}=\omega\left(n^{\beta-2 \alpha}\right)$. In this case, $\rho(m)=\omega\left(\sqrt{\left|S_{t}\right|}\right)$ and $\bar{\Psi}=\omega\left(n^{\beta-2 \alpha}\right)$. Choosing $\rho_{2}$ and $\theta$ in the same way as that in Section IV-A, we can know that infrastructure mode transmissions are the bottleneck compared to ad hoc mode transmissions. Therefore, under bandwidthfairness constraint, the per-node throughput in hybrid mode is as follows:

$$
\begin{aligned}
\widetilde{\lambda}_{H}^{B}(n) & =\frac{\int_{S_{1} \cup S_{2}} \Psi(\xi) \widetilde{B}_{i} \log _{2}(1+S I N R(\xi)) \mathrm{d} \xi}{\int_{\mathcal{A}_{j}} \Psi(\xi) \mathrm{d} \xi} \\
& \geq \frac{\int_{S_{1} \cup S_{2}} \Psi(\xi) \widetilde{B}_{i} \mathrm{~d} \xi}{\int_{\mathcal{A}_{j}} \Psi(\xi) \mathrm{d} \xi} \log _{2}\left(1+\frac{\gamma-2}{2^{\gamma+3}}\left(\frac{\rho(m)}{\rho_{2}}\right)^{\gamma}\right) \\
& \geq \frac{\theta B}{8 \pi \bar{\Psi} \rho^{2}(m)} \log _{2}\left(1+\frac{\gamma-2}{2^{\gamma+3}}\left(\frac{\rho(m)}{\rho_{2}}\right)^{\gamma}\right) .
\end{aligned}
$$

Thus, we can have that when $\theta \rightarrow 1^{-}$,

$$
\begin{aligned}
\widetilde{\lambda}_{H}^{B}(n) & \sim \frac{c_{b} B}{8 \pi \bar{\Psi} \rho^{2}(m)} \log _{2} n \\
& =\frac{n^{\beta-2 \alpha}}{\bar{\Psi}} \cdot \frac{c_{b} B}{800 \beta \ln 2} .
\end{aligned}
$$

where $c_{b}$ equals $c_{t}$ when $\rho(m)=\omega(1), c_{t}^{\prime}$ when $\rho(m)=o(1)$, and $c_{t}^{\prime \prime}$ when $\rho(m)=\Theta(1)$. Comparing (23) with (25), we can see that hybrid mode transmissions can result in higher throughput than pure infrastructure mode transmissions by a scaling factor of $\log _{2} n$. Moreover, comparing (19)-(21) with (25), we can find when using hybrid mode transmissions, bandwidth-fairness can have higher per-node throughput than throughput-fairness by a scaling factor of $\bar{\Psi} / \underline{\Psi}$.

Case II: $\rho(m)=\mathrm{O}(\sqrt{\ln n / \underline{\Psi}})$, i.e., $\underline{\Psi}=\mathrm{O}\left(n^{\beta-2 \alpha}\right)$. In this case, $\pi \rho_{2}^{2}=\mathrm{O}\left(\pi \rho^{2}(m)\right)=\mathrm{O}\left(\left|S_{t}\right|\right)$. Thus, the same as that in Section IV-A, we can obtain that the maximum feasible per-node throughput in hybrid mode is

$$
\widetilde{\lambda}_{H}^{B}(n)=\frac{\underline{\Psi} B}{32 \bar{\Psi}^{2}\left|S_{t}\right|} \log _{2}\left(1+\frac{\gamma-2}{2^{\gamma+3}}\right)=\mathrm{O}\left(\widetilde{\lambda}^{B}(n)\right) .
$$

Thus, when the minimum node density is smaller than the density of base stations in the network, we should carry out all transmissions in infrastructure mode. Moreover, comparing (14) and (23), we can see throughput-fairness and bandwidthfairness lead to the same feasible throughput in this case.

\section{CONCLUSiON}

In this paper, we have investigated the capacity of multihop cellular networks. Both regular and heterogeneous multihop cellular networks are explored, and both throughput-fairness and bandwidth-fairness constraints are considered. We find that compared to traditional cellular networks, regular multihop cellular networks can have higher per-node throughput by a scaling factor of $\log _{2} n$, and heterogeneous cellular networks can achieve the same throughput improvement under certain conditions. Moreover, the results obtained herein also suggest new results for the number of base stations needed to achieve constant per-node throughput in multihop cellular networks.

\section{REFERENCES}

[1] Nokia siemens networks. http://www.nokiasiemensnetworks.com/press/ press-releases/mobile-data-outstrip-voice-traffic-2011, July, 2009.

[2] G. Alfano, M. Garetto, and E. Leonardi. Capacity scaling of wireless networks with inhomogeneous node density: Upper bounds. IEEE Journal on Selected Areas in Communications (JSAC) - Special Issue on Stochastic Geometry and Random Graphs for the Analysis and Design of Wireless Networks, 27(7):1147-1157, September 2009.

[3] CTIA. http://www.ctia.org/advocacy/research/index.cfm/AID/10323, January, 2010.

[4] E. Duarte-Melo, A. Josan, M. Liu, D. Neuhoff, and S. Pradhan. The effect of node density and propagation model on throughput scaling of wireless networks. In Proceedings of IEEE ISIT, Seatlle, Washington, USA, July 2006.

[5] M. Grossglauser and D. Tse. Mobility increases the capacity of ad hoc wireless networks. IEEE/ACM Transactions on Networking, 10(4):477486, August 2002.

[6] P. Gupta and P. Kumar. The capacity of wireless networks. IEEE Transactions on Information Theory, 46(2):388-404, March 2000.

[7] L. Law, S. Krishnamurthy, and M. Faloutsos. Capacity of hybrid cellularad hoc data networks. In Proceeding of IEEE INFOCOM, Phoenix, Arizona, USA, April 2008.

[8] P. Li and Y. Fang. Impacts of topology and traffic pattern on capacity of hybrid wireless networks. IEEE Transactions on Mobile Computing, 8(12):1585-1595, December 2009.

[9] P. Li, C. Zhang, and Y. Fang. Capacity and delay of hybrid wireless broadband access networks. IEEE Journal on Selected Areas in Communications (JSAC) - Special Issue on Broadband Access Networks, 27(2):117-125, February 2009.

[10] Y. Lin and Y. Hsu. Multihop cellular: a new architecture for wireless communications. In Proceeding of IEEE INFOCOM, Tel Aviv, Israel, March 2000.

[11] B. Liu, Z. Liu, and D. Towsley. On the capacity of hybrid wireless networks. In Proceeding of IEEE INFOCOM, San Francisco, California, USA, March 2003.

[12] H. Luo, R. Ramjee, P. Sinha, L. Li, and S. Lu. Ucan: A unified cellular and ad-hoc network architecture. In Proceeding of ACM MobiCom (MobiCom'03), San Diego, CA, USA, June 2003.

[13] T. Rappaport. Wireless Communications: Principles and Practice (Second Edition). Prentice-Hall PTR, 2002.

[14] S. Toumpis. Capacity bounds for three classes of wireless networks. In Proceeding of ACM MobiHoc, Roppongi Hills, Tokyo, Japan, May 2004.

[15] A. Zemlianov and G. Veciana. Capacity of ad hoc wireless networks with infrastructure support. IEEE Journal on Selected Areas in Communications, 23(3), March 2005.

[16] C. Zhang, P. Li, Y. Fang, and P. Khargonekar. Decentralized routing in nonhomogeneous poisson networks. In Proceedings of ICDCS, Beijing, China, June 2008

[17] G. Zhang, Y. Xu, X. Wang, and M. Guizani. Capacity of hybrid wireless networks with directional antenna and delay constraint. IEEE Transactions on Communications, 58(7):2097-2106, 2010. 